\title{
A robotic system to train activities of daily living in a virtual environment
}

\section{Journal Article}

Author(s):

Guidali, Marco; Duschau-Wicke, Alexander; Broggi, Simon; Klamroth-Marganska, Verena; Nef, Tobias; Riener, Robert

Publication date:

2011-10

Permanent link:

https://doi.org/10.3929/ethz-b-000041005

Rights / license:

In Copyright - Non-Commercial Use Permitted

Originally published in:

Medical \& Biological Engineering \& Computing 49(10), https://doi.org/10.1007/s11517-011-0809-0 


\title{
A robotic system to train activities of daily living in a virtual environment
}

\author{
Marco Guidali • Alexander Duschau-Wicke • \\ Simon Broggi • Verena Klamroth-Marganska • \\ Tobias Nef $\cdot$ Robert Riener
}

Received: 18 February 2011/ Accepted: 7 July 2011/Published online: 28 July 2011

(C) International Federation for Medical and Biological Engineering 2011

\begin{abstract}
In the past decade, several arm rehabilitation robots have been developed to assist neurological patients during therapy. Early devices were limited in their number of degrees of freedom and range of motion, whereas newer robots such as the ARMin robot can support the entire arm. Often, these devices are combined with virtual environments to integrate motivating game-like scenarios. Several studies have shown a positive effect of game-playing on therapy outcome by increasing motivation. In addition, we assume that practicing highly functional movements can further enhance therapy outcome by facilitating the transfer of motor abilities acquired in therapy to daily life. Therefore, we present a rehabilitation system that enables the training of activities of daily living (ADL) with the support of an assistive robot. Important ADL tasks have been identified and implemented in a virtual environment. A patient-cooperative
\end{abstract}

Electronic supplementary material The online version of this article (doi:10.1007/s11517-011-0809-0) contains supplementary material, which is available to authorized users.

M. Guidali $(\bowtie) \cdot$ S. Broggi · V. Klamroth-Marganska ·

R. Riener

Sensory-Motor Systems Lab (SMS), ETH Zurich, TAN E,

Tannenstrasse, Zurich, Switzerland

e-mail: marco.guidali@mavt.ethz.ch

M. Guidali · V. Klamroth-Marganska · R. Riener

Spinal Cord Injury Center, Medical Faculty, University Hospital

Balgrist, Forchstrasse 340, Zurich, Switzerland

A. Duschau-Wicke

Hocoma AG, Volketswil, Switzerland

T. Nef

Gerontechnology and Rehabilitation Group, ARTORG

Center for Biomedical Engineering Research,

University of Bern, Bern, Switzerland control strategy with adaptable freedom in timing and space was developed to assist the patient during the task. The technical feasibility and usability of the system was evaluated with seven healthy subjects and three chronic stroke patients.

Keywords Rehabilitation - Robotics - Activities of daily living $\cdot$ Patient-cooperative control

\section{Introduction}

\subsection{Clinical background}

Neurological patients with paralyzed upper extremities (e.g. after stroke) often receive arm therapy to restore motor function, learn new strategies, improve motorcoordination and prevent secondary complications, such as spasticity or joint degeneration. Many studies showed that arm therapy has positive effects on rehabilitation progress [31]. Key factors for an effective rehabilitation are that the training should be intensive [18], repetitive [4], task-oriented [3] and of long duration [37]. Practicing highly functional movements such as those used in activities of daily living (ADL) are assumed to increase the transfer of learned skills to daily life [20].

\subsection{Robot-assisted therapy}

In the last decade, several groups have developed robots for arm therapy. The advantage of these robotic systems is that training duration and intensity can be increased compared to conventional therapy. Furthermore, the robots can measure biomechanical limb functions (e.g. range of motion, torque) and, thus, assess the patient's performance and progress. Many existing robots actuate only single 
degrees of freedom (DoF) or single joints and thus restrict the range of motion of the human arm. Nevertheless, initial clinical studies with devices like the MIT-Manus [16] for planar movements or the Haptic Knob [19] for distal functions have shown that patients can benefit from robotic therapy. Similarly, studies with multi-axial exoskeletal devices for upper extremities, either passive [12] or active $[32,34]$, indicated that stroke patients improve motor control with robot-assisted therapy. Yet, it is still to be proven what the optimal rehabilitation intervention might be. Evidence to date suggests that training the entire arm and the hand at the same time is important to transfer skills from the therapy to daily life $[23,38]$. Accordingly, recent developments in arm rehabilitation devices go towards systems with many DoF to train functional movements. Examples are Pneu-WREX [40], Rupert [36] and Dampace [35] for exoskeleton-based robots and the ADLER [13] and GENTLE/s [22] for end-effector-based devices.

Besides the mechanical constraints to train ADL tasks, environment and control issues have to be considered. The mechanical structure of the robots often impedes interaction with real objects and so most devices are connected to a virtual environment where tasks and feedback are provided. Advantages of virtual environments are the flexibility to switch among different tasks and the motivational aspects of game-like scenarios. Studies found out that motor learning in virtual instead of real environments have some advantages [11]. There is also evidence that skills acquired in a virtual world can be transferred to the real world [11]. Other studies indicate that adding a virtual environment to a rehabilitation robot promotes therapy outcomes [27].

To support the patient during the training, an impedance control strategy that provides active assistance is often used [26]. To maximize the patient's voluntary efforts, the amount of support can be reduced to a minimum using an assist-as-needed (AAN) strategy [8].

This article describes mechanical, environmental, and control components of an novel ADL training system, developed for clinical use. ARMin III was extended with a hand module and combined with a immersive virtual environment to train a variety of different ADL tasks. A control strategy was developed to assist the patient during the training. The feasibility of robot-supported ADL training was evaluated with healthy and stroke subjects as a basis for future clinical studies. The question whether patients can benefit from this type of training was not part of this work, but will be addressed in a subsequent clinical study.

\section{Methods}

ARMin is an arm rehabilitation device developed at ETH Zurich, in collaboration with the University Hospital

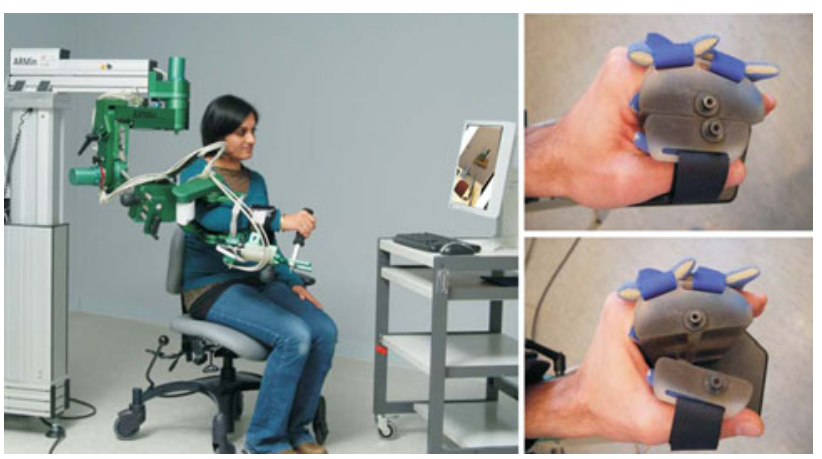

Fig. 1 ARMin III with a subject performing virtual ADL tasks (left) and the hand module (right)

Balgrist. It has an exoskeletal structure with six actuated DoF and an anatomical shoulder actuation [29]. The latest device, version III (Fig. 1), was extended with a hand module to assist grasping. The patient is connected to the robot with cuffs on the upper arm and on the forearm. The hand is fixed into the two handles of the hand module with elastic straps. The lengths of the arm segments, the size of the hand and the height of the device are adjustable to the individual patient.

The range of motion (RoM) of the joints and the nominal torques provided by the actuators are listed in Table 1 . ARMin III can be used for left and right arm training. Each joint has a mechanical end stop to ensure that the anatomical limits of the human arm cannot be exceeded. A spring connected to the upper arm provides passive gravity support as a safety feature in case of a power loss. Redundant position sensors, one analog and one digital sensor for each axis, are used to detect sensor failures and initialize positions during startup.

\subsection{Virtual Environment}

Virtual environments offer a major tool to provide sensory feedback. Users can be immersed in a virtual world and can be motivated with appealing graphics and game play. Recent research indicates that game like tasks can lead to an increased dopamine production, which favors plasticity of the brain [2]. The high flexibility of a virtual environment enables a broad range of tasks that can be trained in a short time and with variation, which enhances the transfer across tasks [14]. A review of Holden et al. [11] found that patients are capable of learning motor tasks in a virtual environment and that the acquired skills can be transferred to the real life. One drawback of virtual training is that realistic tactile feedback at the fingers is difficult to achieve. Another is that perspective in the virtual world can be difficult to establish. To improve the perception of depth, we used appropriate lighting, shadows and special textures. 
Table 1 Technical specification of ARMin III device

\begin{tabular}{lclc}
\hline Axis & RoM & Nom. torque & Gear ratio \\
\hline Arm elevation $q_{1}$ & $40^{\circ}-125^{\circ}$ & $\pm 22.4 \mathrm{Nm}$ & $1: 120$ \\
Plane of elevation $q_{2}$ & $-40^{\circ}-140^{\circ}$ & $\pm 10.5 \mathrm{Nm}$ & $1: 100$ \\
Int./ext. shoulder rotation $q_{3}$ & $-90^{\circ}-90^{\circ}$ & $\pm 12.7 \mathrm{Nm}$ & $1: 144$ \\
Elbow flexion/extension $q_{4}$ & $0^{\circ}-120^{\circ}$ & $\pm 10.5 \mathrm{Nm}$ & $1: 100$ \\
Forearm pro./supination $q_{5}$ & $-90^{\circ}-90^{\circ}$ & $\pm 2.5 \mathrm{Nm}$ & $1: 28.8$ \\
Wrist flexion/extension $q_{6}$ & $-40^{\circ}-40^{\circ}$ & $\pm 6.6 \mathrm{Nm}$ & $1: 233$ \\
Hand opening/closing $q_{7}$ & $0^{\circ}-66^{\circ}$ & $\pm 2.2 \mathrm{Nm}$ & $1: 72$ \\
\hline
\end{tabular}

In our daily routine, we have to execute many different arm movements to ensure a high quality of life. Due to the high range of motion of ARMin, many of those movements would be possible. To reduce the implementation expense, the most important tasks were selected by asking clinical partners, therapists and patients about their preferences. The obtained list consists of 20 ADL tasks like turn a button, open a door or pour water.

The implementation of the virtual ADLs (see Fig. 2) was done with the state-of-the-art game engine GIANTS (GIANTS Software, http://www.giants-software.com) to provide realistic behaviour for objects with the built-in physics and collision engine. Apart from graphical and acoustic rendering, the GIANTS platform acts as a GUI to adjust parameters of ARMin. A second computer running a real-time system (Matlab, xPC Target) controls ARMin with a sampling rate of $1 \mathrm{kHz}$. Communication between the virtual environment and the real time computer is achieved via TCP/IP.

A high-level controller was implemented in the GIANTS engine. Based on the current task and the interaction with the virtual environment, a state machine is updated, and parameters and target positions are sent to the real-time system using the xPC API (Fig. 3). Each target object in the virtual world consists of the end-effector position in space and the orientation of the hand. This target pose is transformed from the virtual to ARMin's coordinate frame. The origin of ARMin's coordinate frame is the glenohumeral joint.

To adjust the virtual tasks to the patient's capabilities (e.g. range of motion, level of difficulty), the location of the avatar in the virtual world can be adjusted with respect to the patient's range of motion.

Feedback about the current target position is given by a small green transparent sphere. Furthermore, instructions are given with text displayed at the top of the screen.

\subsection{Patient-cooperative control}

\subsubsection{Transparency}

In order to obtain satisfactory control performance of patient-cooperative control strategies, the robot should have low friction, negligible backlash and the actuators should be backdrivable [15]. Ideally, a rehabilitation robot is completely transparent, which means that the interaction torque between robot and patient is zero. Due to the mass and friction of the exoskeleton including the actuators, this cannot be fully achieved with a mechanical design. To increase transparency and make the robot more compliant, gravity of the exoskeleton and the safety spring attached to the device have been modeled [29]. Friction of the motorgear combinations has been identified by measurements. A torque $\tau_{\text {comp }}$ summing up these effects is calculated in the control model and used as feed forward compensation.

$\tau_{\text {comp }}=\tau_{g}+\tau_{f}+\left(\tau_{\mathrm{s}}, 0,0,0,0,0,0\right)^{\mathrm{T}}$

The vector $\tau_{\mathrm{g}}$ denotes the gravitational torques for all seven joints, $\tau_{\mathrm{f}}$ the viscous friction torques for all joints and the scalar $\tau_{\mathrm{s}}$ the torque applied by the spring on the arm elevation axis.

\subsubsection{Path control}

Often muscle weakness or ataxia impedes patients' independent performance of a function movement. To assist and support a patient during the training, a patient-cooperative control strategy is required [33]. Such a strategy should maximize the patient's efforts and only assist when and as much as needed.

One strategy proposed for gait and arm rehabilitation is the path control, where the variability of human movements can be accounted for by applying a deadband or virtual tunnel around the desired trajectory [1, 5, 7, 17, 24]. In gait rehabilitation, a normal gait pattern is used as reference. In contrast, the trajectory for arm movements must be calculated taking the current position and the next target position into account. Building a virtual tunnel around this reference trajectory allows the user to move freely within the tunnel, while being guided at the tunnel walls. We implemented a similar strategy for ARMin. Since we aimed to assist the movement of the end-effector position, we developed the algorithm in Cartesian space. A reference trajectory $R$ is determined by the dynamic trajectory 


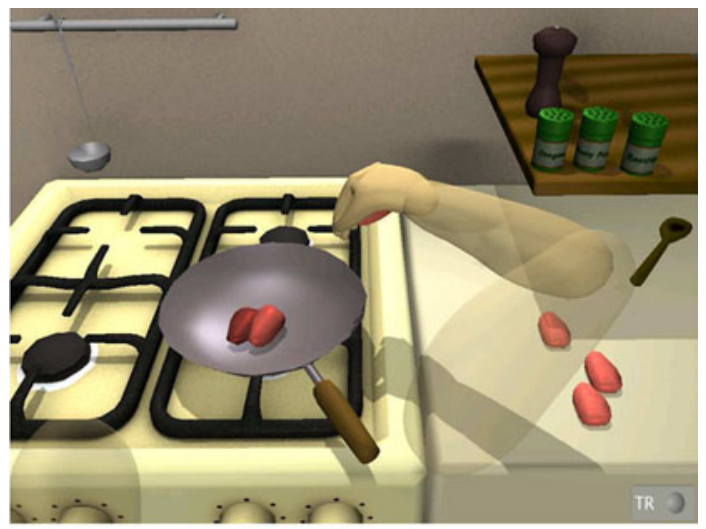

(a) Cooking

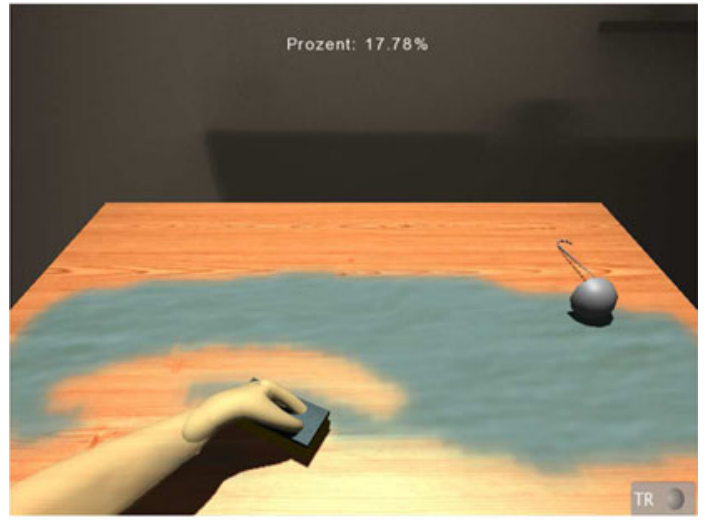

(b) Cleaning

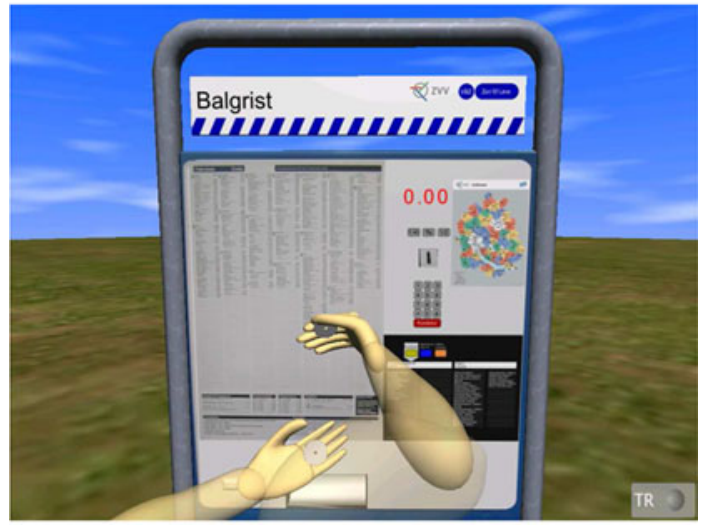

(c) Use a ticket machine

Fig. 2 Three examples of virtual ADL tasks. In a a pan has to be put on the heater and several meatballs must be picked up and dropped into the pan. Task $\mathbf{b}$ involves a dirty table that must be cleaned by moving the hand on a horizontal plane. In task c, coins have to be inserted in a ticket machine

generator (Sect. 2.2.4). For the shape of the tunnel, a cylinder with an adjustable radius $r_{\mathrm{t}}$ was chosen. A nearestneighbor search algorithm is used to calculate the smallest Euclidean distance between the actual position of the endeffector in task space $\mathbf{p}_{\text {act }}$ and the trajectory $R$.

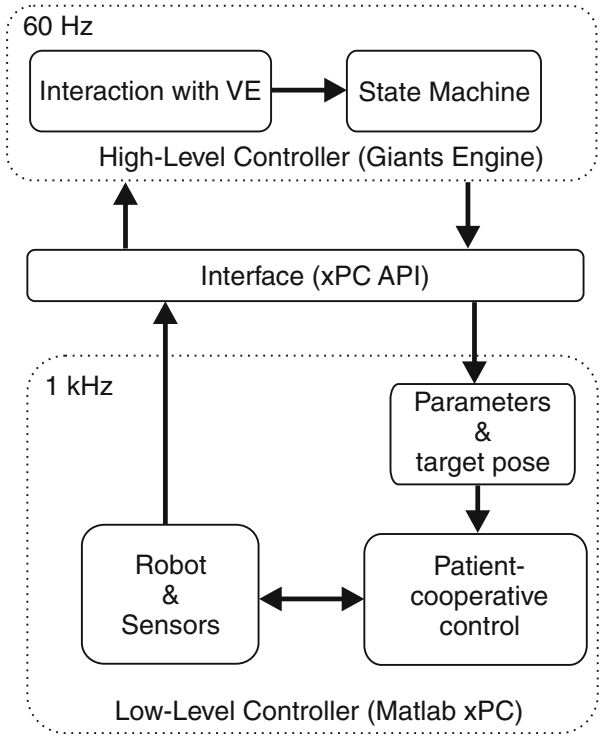

Fig. 3 Controller architecture of the ADL training system

$R:\left\|\mathbf{p}_{\mathrm{ref}}(R)-\mathbf{p}_{\mathrm{act}}\right\|^{2} \stackrel{!}{=} \min$

The resulting trajectory point $\mathbf{p}_{\text {ref }}$ is used as a reference to calculate the supporting forces based on the path-control strategy. A supporting force $\mathbf{F}_{\text {flux }}$ assists the patient along this path like in a flux channel. To determine the direction of the support, we calculate the tangential vector $\mathbf{t}_{\text {flux }}$ at $\mathbf{p}_{\text {ref }}$ along $R$.

$\mathbf{t}_{\text {flux }}=\frac{\mathrm{d}}{\mathrm{d} R} \mathbf{p}_{\text {ref }}(R)$

$\mathbf{F}_{\text {flux }}=k_{\text {flux }} \frac{\mathbf{t}_{\text {flux }}}{\left|\mathbf{t}_{\text {flux }}\right|}$

where $k_{\text {flux }}$ denotes the flux support gain. To ensure that the patient does not get stuck in a position, the amount of support $k_{\text {flux }}$ is adapted during the movement based on a minimally desired mean velocity. A virtual movement is calculated with the selected minimal mean velocity and a bell-shaped velocity profile. If the minimal desired position $p_{\text {min }}$ is ahead of the actual position, $k_{\text {flux }}$ will be increased according to the distance between the two points. The basic flux support $k_{\mathrm{flux}}$ can be adjusted by the therapist in the range of $0-20 \mathrm{~N}$.

$\mathbf{k}_{\text {flux }}= \begin{cases}k_{\text {flux }_{0}} \cdot\left(1+\frac{\left\|p_{\min }-p_{\text {act }}\right\|}{2}\right), & \text { if } p_{\text {min }}>p_{\text {act }} \\ k_{\text {flux }_{0}}, & \text { else }\end{cases}$

Haptic forces are used as guidance along the tunnel wall. Based on a spring-damper system, a force $\mathbf{F}_{\mathrm{w}}$ is generated if the wall is penetrated. The distance $\Delta d$ between actual position and its nearest-neighbor is used to detect a collision between end-effector and virtual tunnel wall. 
$\Delta d=\left(\sum_{i=1}^{3}\left|\mathbf{p}_{\mathrm{ref}}(R)^{(i)}-\mathbf{p}_{\mathrm{act}}^{(i)}\right|^{2}\right)^{\frac{1}{2}}$

$\mathbf{F}_{\mathrm{w}}=\left(K_{\mathrm{w}}\left(\Delta d-r_{\mathrm{t}}\right)+B_{\mathrm{w}} \frac{\mathrm{d}}{\mathrm{d} t}\left(\Delta d-r_{\mathrm{t}}\right)\right) \mathbf{n}$

$\mathbf{n}=\frac{\mathbf{p}_{\mathrm{ref}}(R)-\mathbf{p}_{\mathrm{act}}}{\Delta d}$

with normalized direction $\mathbf{n}$, wall stiffness $K_{\mathrm{w}}$ and walldamping factor $B_{\mathrm{w}}$. The tunnel radius $r_{\mathrm{t}}$ is adjustable in the range of $0-30 \mathrm{~cm}$.

The total force $\mathbf{F}_{\mathrm{pc}}$ which is applied by the path-controller at the end-effector is found by superimposing the two forces.

$\mathbf{F}_{\mathrm{pc}}=\mathbf{F}_{\text {flux }}+\mathbf{F}_{\mathrm{w}}$

The block diagram of the proposed control strategy is shown in Fig. 4.

Arm movements are often point-to-point movements. Therefore, additional constraints at the start and end of the movement are needed to guarantee a safe and determined condition. A force field directed at the target position $\mathbf{p}_{\mathrm{t}}$ closes the path at the end. A similar force field, which opens towards the tunnel entrance, is employed for the start position $\mathbf{p}_{\mathrm{s}}$ (Fig. 5).

Self-initiating a movement is considered essential in motor learning $[21,30]$. To give the patient the possibility of choosing his own timing to start a movement, the following mechanism has been implemented. Whenever the patient has achieved a subtask (e.g. grasped an object), the force field at the end of the tunnel holds the arm at the current position. The patient is now able to initiate the next movement by penetrating the applied force field in the direction of the next target position. The angle $\alpha$ between

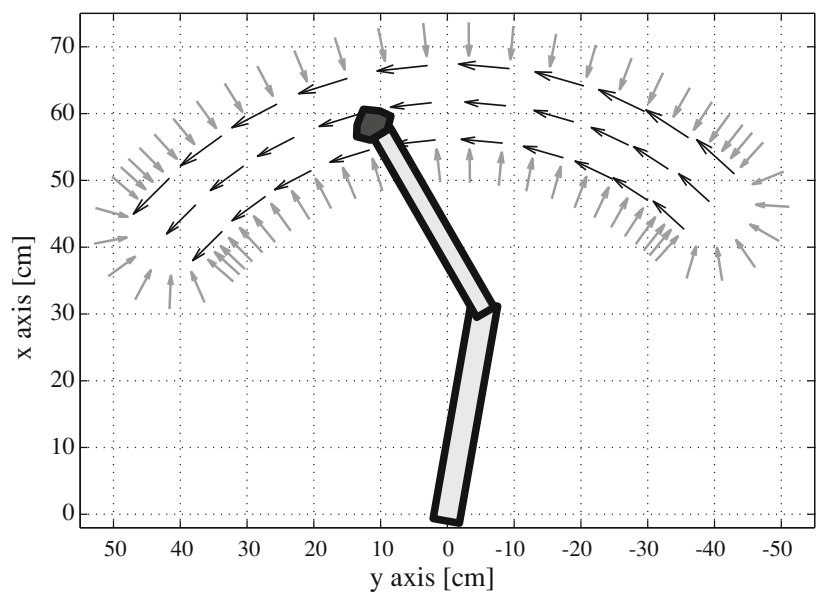

Fig. 5 2-D projection of the force field applied by the path control strategy

the direction of the applied force vector $\mathbf{v}_{\mathrm{af}}$ and the path direction at the beginning of the movement $\mathbf{v}_{\mathrm{p}}$ is used together with a force threshold $F_{\text {init }}$ to trigger the opening of the tunnel toward the next target.

$\mathbf{v}_{\mathrm{af}}=\mathbf{p}_{\mathrm{act}}-\mathbf{p}_{t_{k-1}}$

$\mathbf{v}_{\mathrm{p}}=\frac{\mathrm{d}}{\mathrm{d} R} \mathbf{p}_{s_{k}}$

$\alpha=\arccos \left(\frac{\mathbf{v}_{\mathrm{p}} \cdot \mathbf{v}_{\mathrm{af}}}{\left|\mathbf{v}_{\mathrm{p}}\right|\left|\mathbf{v}_{\mathrm{af}}\right|}\right)$

$T_{\text {init }}= \begin{cases}1, & \text { if }|F|>F_{\text {init }} \& \alpha<\alpha_{\text {init }} \\ 0, & \text { otherwise }\end{cases}$

with $T_{\text {init }}$ as trigger to open the tunnel.

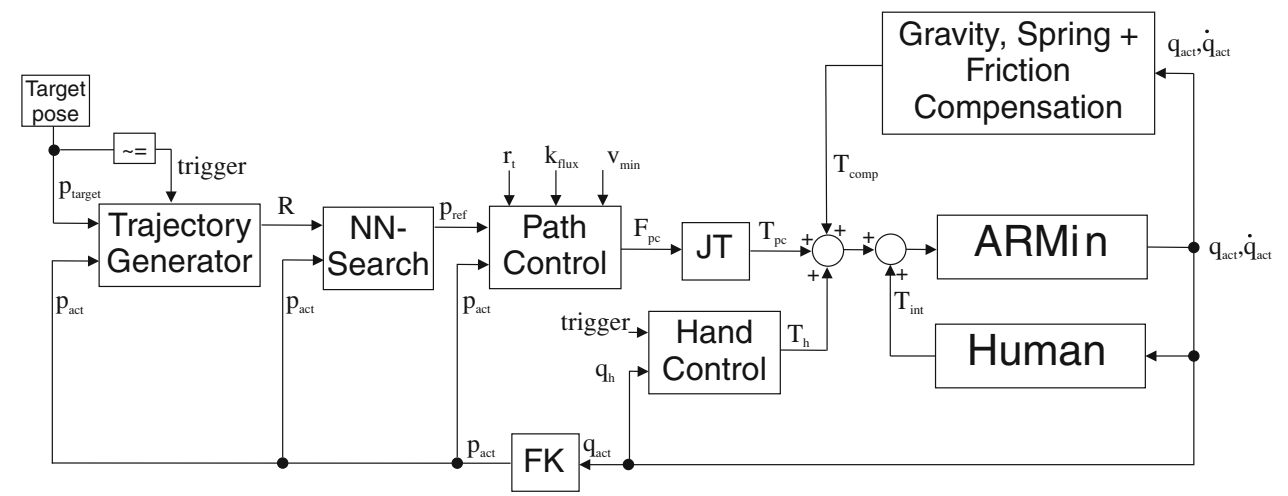

Fig. 4 Block diagram of path control and hand control strategy. If a new target pose is received, the "Trajectory Generator" is triggered and calculates a new trajectory, $R$, based on the actual position in space and the target position. With the "NN-Search", the closest point on the trajectory is determined and used as reference $\mathbf{p}_{\text {ref }}$ for the path controller, that calculates the supportive and guidance forces that are applied to the robot (Eqs. 4 and 7). The hand control is triggered by the the high level controller and assists the hand function with an impedance controller. In addition, a torque $\tau_{\text {comp }}$ is added to compensate for gravity, friction and the passive spring of ARMin. The interaction torque $\tau_{\text {int }}$ is applied by the human and cannot be measured directly 


\subsubsection{Hand control}

Arm movements can be supported by the path control strategy described above. The function of the hand is assisted by a triggered position controller. Once the target position is reached, the high-level controller of the virtual environment (see Sect. 2.1) sends commands to the hand module that depends on three possible states: grasp object, drop object or hold object. If an interaction occurs (e.g. object dropped), the support of the hand is turned off. This allows free movement of the hand during the arm movement. In addition, if a patient with hand spasticity is trained, he should be able to go back to a comfortable position after opening his hand with assistance of the robot.

As reference for the impedance controller, a trajectory is generated based on the minimal jerk method and with a mean velocity of $5 \%$ s. The desired angle $q_{h_{\text {des }}}$ for the controller follows this trajectory. To allow faster movements, the controller works only unidirectionally. If the actual hand position is in front of the desired position, the supportive hand torque $\tau_{\mathrm{h}}$ is set to zero.

$\tau_{\mathrm{h}}=K_{\mathrm{h}}\left(q_{h_{\mathrm{des}}}-q_{h_{\mathrm{act}}}\right)+B_{\mathrm{h}}\left(\dot{q}_{h_{\mathrm{des}}}-\dot{q}_{h_{\mathrm{act}}}\right)$

The impedance $K_{\mathrm{h}}$ of the controller is adjustable by the therapist between $0-0.27 \frac{\mathrm{Nm}}{\circ}$. The damping factor $B_{\mathrm{h}}$ is selected, so that the ratio of $\frac{K_{\mathrm{h}}}{B_{\mathrm{h}}}$ stays constant [6].

\subsubsection{Trajectory generation}

To assist and support the patient with the path-control strategy, a reference trajectory is required. Due to the huge variability in start and target position, a mathematical description of the arm trajectory is indispensable. Different reach and grasp studies showed, for instance, that movements in the midsagittal plane are less curved than movements from left to right [10]. We investigated in a previous study different trajectory generation methods for the ADL tasks with ARMin and found that the minimum angular jerk method is superior to other common methods like e.g. minimal jerk [9]. By minimizing the change of angular acceleration the trajectory is given by

$C: \frac{1}{2} \int_{0}^{t_{f}} \sum_{i=1}^{n}\left(\frac{d^{3} q_{i}}{\mathrm{~d} t^{3}}\right)^{2} \mathrm{~d} t \stackrel{!}{=} \min$

Representing this formula as a fifth order polynomial allows us to create an online computation of the problem.

$q(t)=a_{0}+a_{1} t+a_{2} t^{2}+a_{3} t^{3}+a_{4} t^{4}+a_{5} t^{5}$

Assuming that velocity and acceleration are zero at the beginning and the end of the trajectory, we can use the following equation for the minimum angular jerk trajectory: $\mathbf{q}(t)=\mathbf{q}_{\mathrm{s}}+\mathbf{l}\left(10\left(\frac{t}{t_{\mathrm{f}}}\right)^{3}-15\left(\frac{t}{t_{\mathrm{f}}}\right)^{4}+6\left(\frac{t}{t_{\mathrm{f}}}\right)^{5}\right)$

$\mathbf{l}=\mathbf{q}_{\mathrm{t}}-\mathbf{q}_{\mathrm{s}}$

where $\mathbf{q}_{\mathrm{s}}$ is the start position, $\mathbf{q}_{\mathbf{t}}$ the target position and $\mathbf{l}$ is the vector between the two in joint space. The variable $t_{\mathrm{f}}$ denotes the duration of the movement. To decouple the trajectory from the time information, we can replace the time information $t$ and $t_{\mathrm{f}}$ with discrete points $k$ and total number of points $k_{\mathrm{f}}$ on the trajectory.

$\mathbf{q}(k)=\mathbf{q}_{\mathrm{s}}+\mathbf{l}\left(10\left(\frac{k}{k_{\mathrm{f}}}\right)^{3}-15\left(\frac{k}{k_{\mathrm{f}}}\right)^{4}+6\left(\frac{k}{k_{\mathrm{f}}}\right)^{5}\right)$

By applying the forward kinematics to the trajectory in joint space $\mathbf{q}(k)$, we get the required reference trajectory $\mathbf{p}(\mathrm{k})$ for the end-effector in task space.

\subsection{Validation}

Two experiments have been conducted to validate the system. In the first experiment, healthy subjects performed the virtual ADLs with ARMin to test proper function and safety of the system. Healthy subjects should be able to move freely within the virtual tunnel and carry out the tasks demanded without being hindered by the robot. In the second experiment, stroke patients trained the ADLs with ARMin to investigate whether it would be clinically feasible to use such a system in patients and how well the patients would be able to perform ADLs compared to healthy subjects. Both, patients and healthy subjects, received written and verbal information about the study by the therapist and gave written informed consent. The study protocol meets the institutional guidelines and was approved by the institutional committees (Swissmedic and Cantonal Ethics Committee Zurich).

The virtual environment and the robot were operated and controlled by a skilled therapist. During both experiments the robot recorded sensory data, the task to be carried out and forces applied by the path controller. ARMin's transparency was improved using gravity, spring and viscous friction compensation (see Sect. 2.2.1). The path could be visualized in the virtual world by green rings used to show the subjects the idea of the tunnel before the experiment. To become familiar with the robot and the virtual task all subjects did the whole ADL task once before the experiment.

\subsubsection{Experiment with healthy subjects}

Seven healthy subjects participated in the experiment (four female, three male; mean age 34.3 years, SD 9.4). Before 
the experiment, all subjects were informed about the ARMin setup. After becoming familiar with the device and the virtual world, they had to perform one specific ADL task consisting of 16 reach-and-grasp movements in a virtual kitchen (Fig. 2a). First, the pan had to be grasped and put on the burner. Then, the seven meatballs had to be picked up and dropped into the pan. The target position was always indicated by a small transparent green sphere. While the drop position above the pan was constant, the pick-up position was randomly chosen among the meatballs remaining on the table.

The path control strategy was applied, but with zero support force inside the tunnel $F_{\text {flux }}=0 N$. Preliminary tests showed that a tunnel radius of $r_{\mathrm{t}}=8 \mathrm{~cm}$ is appropriate for most subjects. The parameters for the haptic tunnel wall were selected with a trial and error method with the goal to achieve a soft interaction between virtual wall and endeffector. A wall stiffness of $K_{\mathrm{w}}=250 \mathrm{~N} / \mathrm{m}$ and a wall damping of $B_{\mathrm{w}}=50 \mathrm{Ns} / \mathrm{m}^{2}$ were used to achieve this. No hand support was provided by the hand controller.

In a second setup, the intention detection method was evaluated. With the goal to find a minimal value for the force barrier $F_{\text {init }}$ to initiate a movement correctly, five values between 0.1 and $5 \mathrm{~N}$ were tested. The angle $\alpha_{\text {init }}$ was set to $45^{\circ}$.Initiation should only be detected if the subject voluntarily applied a force. When the algorithm detected an intention the force flux inside the tunnel started to support the movement, so that the subjects could rate whether the initiation was voluntary or not. In addition, the subjects had to state if they could feel the initiation barrier. The experiment was conducted ten times for the selected force threshold values.

\subsubsection{Experiment with stroke subjects}

Three chronic stroke subjects were recruited to test the system (Table 2). All three patients already had experience with the device due to an ongoing study and had recently undergone a test battery including the Fugl-Meyer Assessment (FMA, upper extremity motor part). An occupational therapist adjusted the device to the patient and instructed the patient before and during the experiment. The task and the virtual wall stiffness $K_{\mathrm{w}}$ and damping $B_{\mathrm{w}}$ were the same as for healthy subjects described in the first experiment. The basic flux support $k_{\mathrm{flux}_{0}}$ was adjusted by the therapist to the patient's capabilities. Starting with zero support, the gain was increased until the patient could solve the demanded task. To start the movement, the described intention detection algorithm was used. After a $5 \mathrm{~s}$ waiting time, the robot initiated the next movement, if no movement intention was detected. The hand was supported with the described triggered impedance controller with $K_{\mathrm{h}}=0.02-0.08 \frac{\mathrm{Nm}}{\circ}$.

After the experiment, the patients had to rate their motivation on a scale of one to five where 1 means not motivated at all and 5 very motivated. The therapists were asked if the visual aids were helpful to adjust the controller.

\section{Results}

\subsection{Experiment with healthy subjects}

All healthy subjects understood the task clearly and were able to perform the requested movements to achieve the goal. During the task, movements of subjects were constrained to the virtual tunnel in task space created by the path controller (Fig. 6).

The mean and peak speed of the end-effector during the movements was calculated as $24.94 \mathrm{~cm} / \mathrm{s}(\mathrm{SD}=5.88 \mathrm{~cm} / \mathrm{s})$ and $56.44 \mathrm{~cm} / \mathrm{s}(\mathrm{SD}=9.63 \mathrm{~cm} / \mathrm{s})$, respectively (see Fig. 7). Total time to finish the task, including the interaction with

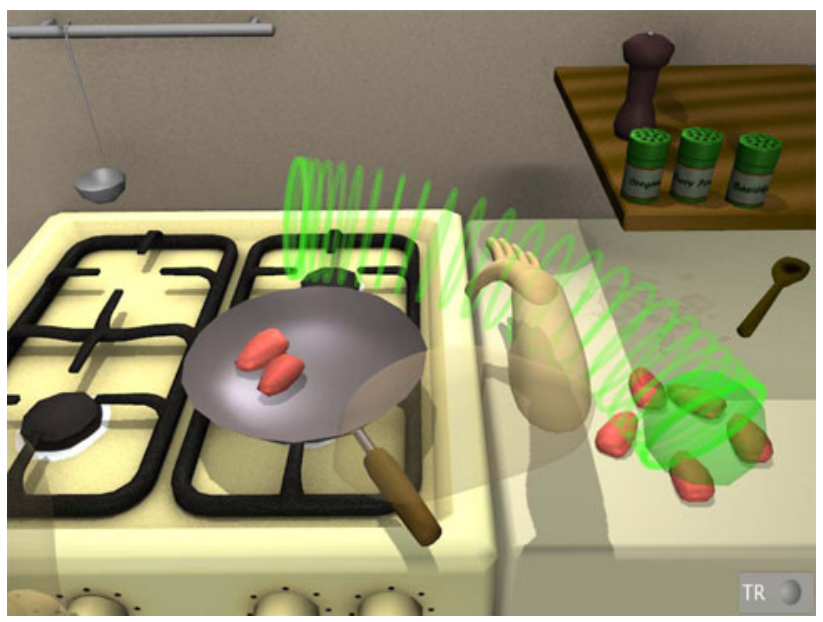

Fig. 6 Kitchen scenario with visualized path (green rings). The green sphere at the end of the path indicates the target position to grasp the next meatball

Table 2 Patient characteristics

\begin{tabular}{llllll}
\hline Subject no. & Sex & Age & Affected side & Months post-stroke & FMA \\
\hline P1 & f & 64 & L & 84 & 26 \\
P2 & m & 61 & L & 19 & 26 \\
P3 & f & 34 & R & 18 & 11 \\
\hline
\end{tabular}




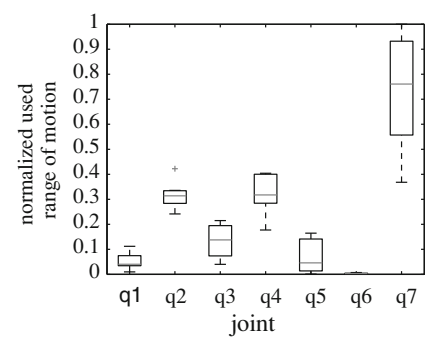

(a)Healthy

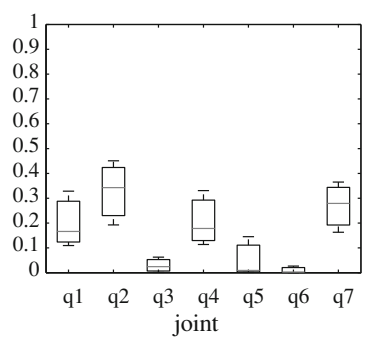

(b)Patients
Fig. 7 Mean used relative range of motion by the seven healthy subjects (a) and the three stroke patients (b) to solve the task. The corresponding joint names are listed in Table 1

objects, varied significantly among the subjects and was on average $44.69 \mathrm{~s}(\mathrm{SD}=22.94 \mathrm{~s})$. The mean task duration was divided into a reaching and a grasping segment and is shown in Fig. 7. Examples of performed trajectories can be found in Online Resource 2.

To evaluate which joints were mainly involved to solve the task the mean relative range of motion for all seven joints was calculated for each subject and normalized with the total range of motion. The mean values over all subjects are depicted in Fig. 8 and show that mainly the horizontal shoulder joint $\left(q_{2}\right)$, the elbow joint $\left(q_{4}\right)$ and the hand $\left(q_{7}\right)$ had to be used to solve the task. This was expected as the task did not require excessive arm elevation $\left(q_{1}, q_{3}\right)$ or change of the hand orientation $\left(q_{3}, q_{5}\right)$. The inter-subject variation shows that the path controller allowed the subjects to choose their own trajectory. A summarizing table with individual subject results can be found in Online Resource 1.

When touching the virtual tunnel wall, a force to prevent penetration was applied by the path controller. The time of wall contact was measured during each movement and normalized with the time needed to cross the tunnel. On average, the healthy subjects were touching the walls $54.0 \%$ ( $\mathrm{SD}=17.5 \%)$ of the time. Investigating the induced force vector shows that the tunnel was mainly penetrated at the bottom [mean $\mathrm{F}_{x}=1.8 \mathrm{~N}(\mathrm{SD}=1.6 \mathrm{~N})$, mean $F_{y}=$ $1.1 \mathrm{~N}(\mathrm{SD}=0.8 \mathrm{~N})$, mean $\left.F_{z}=3.4 \mathrm{~N}(\mathrm{SD}=2.8 \mathrm{~N})\right]$.

Table 3 shows the result of the intention detection evaluation. The number of true positive (TP) and the number of false positive (FP) were counted to evaluate the accuracy $(\mathrm{ACC}=\mathrm{TP} /(\mathrm{TP}+\mathrm{FP}))$ of the algorithm . For the subsequent experiments with stroke patients, a value of $1 \mathrm{~N}$ was chosen for the force threshold.

\subsection{Experiment with stroke subjects}

Three chronic stroke patients have used the system to train ADL in the virtual environment. All patients understood the tasks to do. Assistance for arm movements was provided by the path-control strategy, where the basic amount of support was adjusted to the capabilities of the patient. With the force flux turned off, the patients could not do the required movements. Subject P2 was able to do the task with a flux support of $k_{\text {flux }_{0}}=2.0 \mathrm{~N}$. The other two patients, $\mathrm{P} 1$ and $\mathrm{P} 3$, needed a value of $k_{\mathrm{flux}_{0}}=4.5 \mathrm{~N}$ and $4.0 \mathrm{~N}$, respectively, to perform the task. When the patient was slower than the given minimal desired movement, this value was increased by the controller according to Eq. 5 (Fig. 9). The mean recorded flux support for subject P1 was $6.6 \mathrm{~N}(\mathrm{SD}=4.8 \mathrm{~N})$, for $\mathrm{P} 22.0 \mathrm{~N}(\mathrm{SD}=0.0 \mathrm{~N})$ and for $\mathrm{P} 3$ $5.8 \mathrm{~N}(\mathrm{SD}=1.2 \mathrm{~N})$. With the selected impedance for the hand controller $\left(K_{\mathrm{h}}=0.02-0.08 \frac{\mathrm{Nm}}{\mathrm{o}}\right)$ all patients were able to open and close their hand.

The mean and peak speeds of the three stroke subjects were $21.1 \mathrm{~cm} / \mathrm{s}(\mathrm{SD}=8.97 \mathrm{~cm} / \mathrm{s})$ and $46.0 \mathrm{~cm} / \mathrm{s}(\mathrm{SD}=$ $13.34 \mathrm{~cm} / \mathrm{s}$ ), respectively. On average, patients needed $157.49 \mathrm{~s}(\mathrm{SD}=113.82 \mathrm{~s})$ to finish the whole task. The boxplots of those measures are shown in Fig. 7. As for the healthy subjects, the mean relative joint motion was calculated for each subject (see Fig. 8). Horizontal shoulder movement $\left(q_{2}\right)$, elbow flexion and extension $\left(q_{4}\right)$ and hand opening and closing $\left(q_{7}\right)$ were the dominant joint actions involved in the task. Even though the patients were assisted

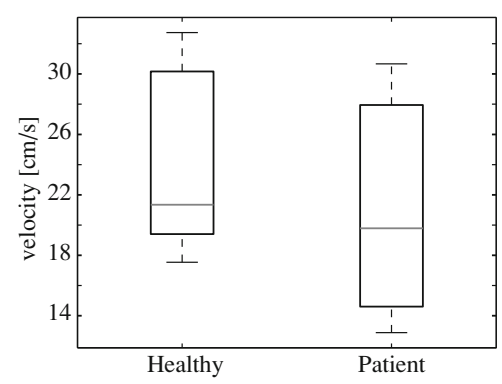

(a) mean velocity

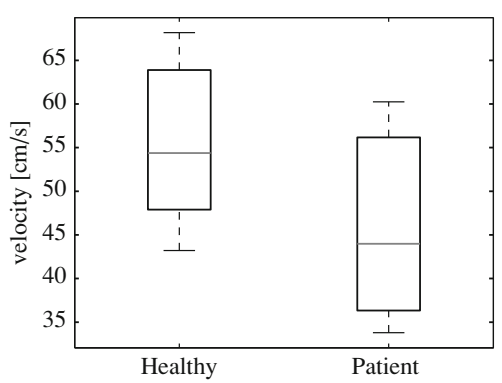

(b) peak velocity

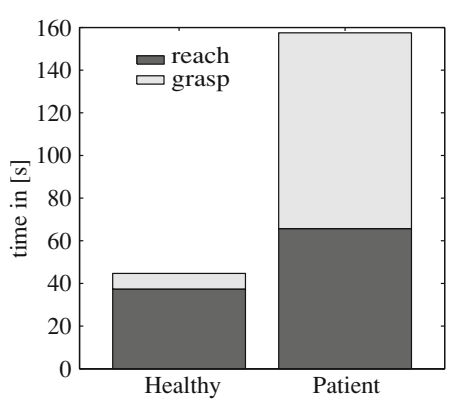

(c) task execution time

Fig. 8 a Boxplot of mean velocity of end-effector, $\mathbf{b}$ boxplot of peak velocity of end-effector and $\mathbf{c}$ task execution time split up in a reach and a grasp part for the seven healthy subjects and the three stroke patients 
Table 3 Intention detection evaluation to find an optimal value for the force barrier $F_{\text {init }}$. With an increasing force barrier the number of wrong detections decreases, but the higher the chance that the the subject perceives this force threshold, which is not desired

\begin{tabular}{|c|c|c|c|c|c|c|}
\hline \multirow[b]{2}{*}{$F_{\text {init }}$} & \multicolumn{3}{|c|}{ Detection accuracy } & \multicolumn{3}{|c|}{ Force barrier perceived } \\
\hline & $\mathrm{TP}$ & FP & $\%$ & Yes & No & $\%$ \\
\hline 0.1 & 33 & 37 & 47.14 & 0 & 70 & 0 \\
\hline 0.5 & 50 & 20 & 71.43 & 0 & 70 & 0 \\
\hline 1 & 70 & 0 & 100.0 & 3 & 67 & 4.29 \\
\hline 2 & 70 & 0 & 100.0 & 10 & 60 & 15 \\
\hline 5 & 70 & 0 & 100.0 & 53 & 70 & 75.5 \\
\hline
\end{tabular}

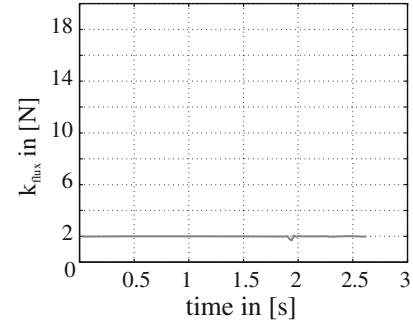

(a)

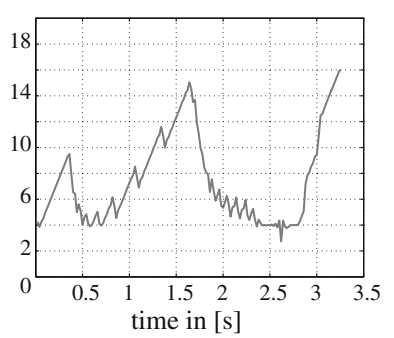

(b)
Fig. 9 Example of the adaptation of the flux gain $k_{\text {flux }}$ by the controller based on the minimal desired performance. Both patients did the same movement. a Flux gain of subject P2 and $\mathbf{b}$ Flux gain of subject P1

by the force flux, the variation in joints used shows that the patients could choose their own trajectory as desired by our control paradigm.

Looking at the interaction forces between the endeffector and the tunnel wall of the path controller showed that during $42.4 \%$ ( $\mathrm{SD}=17.8 \%$ ) of the movement, patients were touching the walls generating the forces $F_{x}=$ $-0.87 N(\mathrm{SD}=1.00 \mathrm{~N}), F_{y}=-0.13 N(\mathrm{SD}=0.90 \mathrm{~N})$ and $F_{z}=0.78 N(\mathrm{SD}=0.96 \mathrm{~N})$.

With the found parameters for the initialization algorithm, all patients were able to decide when to start a movement. The wait time of $5 \mathrm{~s}$ was never reached.

All patients rated their motivation with a five out of five possible points and reported that they would like to train with such a system. The three therapists stated that the visual aids helped them to adjust the cooperative-controller.

\section{Discussion}

\subsection{Patient-cooperative control}

Transparency of the device was improved by compensating for gravity, coulomb friction and the spring used for passive gravity compensation with the actuators. Even though healthy subjects could easily move the robot, the inertia of the exoskeleton and the static friction of the actuators impeded full transparency of the device. By adding the recently developed static friction compensation method, we could further improve the transparency [28]. Extending the robot with acceleration sensors to the robot would be needed to also compensate for inertia. The path control strategy proved to be a good approach to support the patient during point-to-point movements. However, it cannot be used to support movements where mainly hand orientation is involved (e.g. during turning a button). To cover all possible tasks other strategies have to be investigated and integrated into the system.

\subsection{Comparing performance of healthy and stroke subjects during ADL training}

Both healthy and stroke subjects were able to use ARMin for ADL training. Patients who performed the movements with the assistance of the path-control strategy achieved almost the same mean velocity as the healthy subjects without support. However, the mean total execution time for the task was almost four times higher in patients than in healthy subjects. Dividing the task execution time into reach and grasp segments showed that the time required to grasp the objects contributed most to this huge difference. There are two potential reasons for that effect. First, patients had more problems coordinating their hand position to grasp an object probably because perception of the virtual world-implemented in $3 \mathrm{D}$, but displayed on a $2 \mathrm{D}$ monitor-is weaker in stroke survivors than in healthy subjects. Second, no stroke survivor could open his hand by himself, so all were highly dependent on the support of the robot to assist them in opening the hand. Due to safety considerations (e.g. spastic hand), the speed of the hand opening was set to $5 \%$, so that $3-5 \mathrm{~s}$ were required to open a very weak or spastic hand.

As desired, the spatial freedom given by the path controller allowed the subjects to use their own trajectory. Therefore, trick movements or compensatory strategies of patients are possible to some degree.

Unexpectedly, the interaction forces with the virtual tunnel were smaller in patients than in healthy subjects. 
Asking healthy subjects after the experiment whether they had felt the tunnel wall during the task, only two stated that they felt them in 1 out of the 16 movements. This, and the fact that the main direction of the tunnel interaction force was in gravitational direction (z-direction), suggests that the healthy subjects used the tunnel wall to minimize their efforts using the tunnel wall as a sort of gravity support. Another argument is that the target position to drop the meatballs was maybe set too high above the pan and the subjects tried to drop the objects as close to the pan as possible to minimize their effort in lifting their arm. To prevent the subjects from learning to rely too much on the wall support, visual, auditory or haptic feedback should be added to inform them.

\subsection{Feasibility of ADL training with stroke subjects}

The proposed system was designed to create an interactive and realistic training setup for functional tasks that can be used in stroke rehabilitation. Three skilled therapists adjusted the device and used the GUI to set individual parameters for patients. They also positioned patients and gave instructions about tasks. The visual aids to explain the path-control strategy seemed to help the therapists understand the meaning of the parameters.

All tested stroke patients were very eager to train with the system. They also orally confirmed the impression that they were very motivated to do ADL training with ARMin after the experiment. The applied assistance helped them to do functional movements, which they could not do otherwise. The amount of support was adapted by the controller based on a minimally desired movement speed. Although patients P1 and P2 had the same score in the FMA, the amount of support needed to achieve the same task was quite different. This was not so surprising when looking at the build of the two patients. P1 was a slim female and P2 a well-built male, which could produce much more force than P1. Force production is only a small portion of the FMA and also the distribution of the points within the FMA can be completely different, while still leading to the same score. Further studies with more patients are needed to investigate if there is a correlation between FMA, force and required arm support during ADL. In addition, a method to measure and display feedback about the patient's participation like proposed by Lunenburger et al. [25] would be beneficial to prevent the patient from starting to rely too much on the adaptive support after a few trials.

\subsection{Limitations}

Training for several ADL tasks with a rehabilitation robot in a realistic way requires a complex system. Ideally, the patient should be able to decide what movements or tasks he wants to do and the robot would detect those intentions and assist him as needed. To limit complexity, we decided to predefine the sequence of the tasks. This reduced flexibility may give the patient the feeling that he is not fully in charge. An extension of the algorithm to choose among different possible targets or a combination with a sophisticated BCI [39] could improve the interaction between patient, robot and virtual world. Although the virtual tasks were implemented in a realistic way, some healthy subjects stated that the scenario of sitting in a kitchen in front of the heater felt unnatural.

The adaptation of the support is based on a minimal desired velocity of movement and the initial amount of support is chosen by the therapist. An extension of the path controller with an algorithm proposed by Wolbrecht et al. [40], which learns the amount of arm support needed online would further improve the adaptation of the strategy to the patient's capabilities.

Our training paradigm requires a complex rehabilitation system consisting of mechanical design, control method and a virtual environment. In case of a possible effect on therapy outcome, we will not be able to assign it to the single components.

However, it could be shown that the developed training system enables the training of virtual ADL for stroke survivors. As the technical and clinical feasibility of the system could be proven, a controlled clinical multicenter study has been started, where 80 patients receive either conventional or robotic training, to investigate the effects on therapy outcome.

Acknowledgements The authors would like to thank the healthy subjects and especially the patients who participated in this study. We also thank the therapists Anja Kollmar, Olivier Schmid and Daniela Kammerer who trained the patients and gave helpful input and feedback. Furthermore, we thank the Hocoma AG, Volketswil, Switzerland, for their contribution to the development of the hand module. The project was supported by the Swiss Research Foundation NCCR on Neural Plasticity, the Swiss National Science Foundation through the National Centre of Competence in Research Robotics and by the Swiss National Science Foundation (SNF) grant 325200-1260621.

\section{References}

1. Banala SK, Agrawal SK, Scholz JP (2007) Active leg exoskeleton (ALEX) for gait rehabilitation of motor-impaired patients. In: Proceedings of the IEEE international conference on rehabilitation Robotics, Noordwijk, pp 401-407

2. Bavelier D, Levi DM, Li RW, Dan Y, Hensch TK (2010) Removing brakes on adult brain plasticity: from molecular to behavioral intervention. J Neurosci 45(30):14964-14971

3. Bayona NA, Bitensky J, Salter K, Teasell R (2005) The role of task specific training in rehabilitation therapies. Top Stroke Rehabil 12:58-65

4. Butefisch C, Hummelsheim H, Denzler P, Mauritz KH (1995) Repetitive training of isolated movements improves the outcome 
of motor rehabilitation of the centrally paretic hand. J Neurol Sci 130:59-68

5. Cai LL, Fong AJ, Otoshi CK, Liang Y, Burdick JW, Roy RR, Edgerton VR (2006) Implications of assist-as-needed robotic step training after a complete spinal cord injury on intrinsic strategies of motor learning. J Neurosci 26(41):564-568

6. Colgate JE, Brown JM (1994) Factors affecting the Z-width of haptic display. In: Proceedings of the international conference on Robotics and Automation, San Diego, pp 3205-3210

7. Duschau-Wicke A, von Zitzewitz J, Caprez A, Lnenberger L, Riener R (2009) Path control: a method for patient-cooperative robot-aided gait rehabilitation. IEEE Trans Neural Syst Rehabil Eng 18(1):38-48

8. Emken JL, Bobrow JE, Reinkensmeyer DJ (2005) Robotic movement training as an optimization problem: designing a controller that assists only as needed. In: Proceedings of the IEEE 9th international conference rehabilitation Robot, pp 307-312

9. Guidali M, Bchel M, Klamroth V, Nef T, Riener R (2009) Trajectory planning in ADL tasks for an exoskeletal arm rehabilitation robot. In: Proceedings of the European conference on technically assisted rehabilitation, Berlin, pp 20-24

10. Haggard P, Richardson J (2003) Spatial patterns in the control of human arm movement. Exp Psychol 2:42-62

11. Holden MK (2005) Virtual environments for motor rehabilitation: review. CyberPsychol Behav 3(8):187-211

12. Housman SJ, Scott KM, Reinkensmeyer DJ (2009) A randomized controlled clinical trial of gravity-supported, computer-enhanced arm exercise for individuals with severe hemiparesis. Neurorehabil Neural Repair 23(5):505-514

13. Johnson MJ, Wisneski KJ, Anderson J, Nathan D, Smith RO (2006) Development of ADLER: the activities of daily living exercise robot. In: Proceedings of the biomedical Robotics and Biomechatronic, pp 881-886

14. Kornell N, Bjork RA (2008) Learning concepts and categories: is spacing the enemy of induction. Psychol Sci 19(6):585-592

15. Krebs HI (2004) Rehabilitation robotics: pilot trial of a spatial extension for MIT-Manus. J Neuroeng Rehabil 1(5):1-5

16. Krebs HI, Hogan N, Volpe BT, Aisen ML, Edelstein L, Diels C (1999) Overview of clinical trials with MIT-MANUS: a robotaided neuro-rehabilitation facility. Technol Health Care 7:419-423

17. Krebs HI, Palazzolo JJ, Dipietro L, Ferraro M, Krol J, Rannekleiv K, Volpe BT, Hogan N (2003) Rehabilitation robotics: performance-based progressive robot-assisted therapy. Auton Robots 15(1):7-20

18. Kwakkel G, Wagenaar RC, Koelman TW, Lankhorst GJ, Koetsier JC (1997) Effects of intensity of rehabilitation after stroke. A research synthesis. Stroke 28:1550-1556

19. Lambercy O, Dovat L, Gassert R, Burdet E, Teo CL, Milner T (2007) A haptic knob for rehabilitation of hand function. IEEE Trans Neural Syst Rehabil Eng 3(15):356-366

20. Langhammer B, Stanghelle JK (2000) Bobath or motor relearning programme? A comparison of two different approaches of physiotherapy in stroke rehabilitation: a randomised controlled study. Clin Rehabil 14:361-369

21. Lotze M, Braun C, Birbaumer N, Anders S, Cohen LG (2003) Motor learning elicited by voluntary drive. Brain Behav Evol 126(4):866-872

22. Loureiro RCV, Harwin WS (2007) Reach \& grasp therapy: design and control of a 9-DOF robotic neuro-rehabilitation system. In: Proceedings of the international conference on rehabilitation Robotics, Kyoto, pp 757-763

23. Lum P, Reinkensmeyer D, Mahoney R, Rymer WZ, Burgar C (2002) Robotic devices for movement therapy after stroke: current status and challenges to clinical acceptance. Top Stroke Rehabil 8(4):40-53

24. Lum P, Burgar CG, Shor PC, Majmundar M, van der Loos M (2004) Evidence for improved muscle activation patterns after retraining of reaching movements with the MIME robotic system in subjects with post-stroke hemiparesis. IEEE Trans Neural Syst Rehabil Eng 12(2):186-194

25. Lunenburger L, Colombo G, Riener R, Dietz V (2004) Biofeedback in gait training with the robotic orthosis Lokomat. Eng Med Biol Soc 2:4888-4891

26. Marchal-Crespo L, Reinkensmeyer DJ (2009) Review of control strategies for robotic movement training after neurologic injury. J NeuroEng Rehabil 6(20):20

27. Mirelman A, Bonato P, Deutsch JE (2009) Effects of training with a robot-virtual reality system compared with a robot alone one the gait of individuals after stroke. Stroke 40(1):169-174

28. Nef T, Lum P (2009) Improving backdrivability in geared rehabilitation robots. Med Biol Eng Comput 47(4):441-447

29. Nef T, Guidali M, Riener R (2009) ARMin III-arm therapy exoskeleton with an ergonomic shoulder actuation. Appl Bionics Biomech 6(2):127-142

30. Perez MA, Lungholt BK, Nyborg K, Nielsen JB (2004) Motor skill training induces changes in the excitability of the leg cortical area in healthy humans. Exp Brain Res 159(22):197-205

31. Platz T (2003) Evidence-based arm rehabilitation-a systematic review of the literature [publication in German]. Nervenarzt 74:841-849

32. Prange GB, Jannink MJA, Groothuis-Oudshoorn CGM, Hermens HJ, IJzerman MJ (2006) Systematic review of the effect of robotaided therapy on recovery of the hemiparetic arm after stroke. J Rehabil Res Dev 43(2):171-184

33. Riener R, Lunenburger L, Jezernik S, Anderschitz M, Colombo G, Dietz V (2005) Patient-cooperative strategies for robot-aided treadmill training: first experimental results. IEEE Trans Neural Syst Rehabil Eng 13(3):380-394

34. Staubli P, Nef T, Klamroth-Marganska V, Riener R (2009) Effects of intensive arm training with the rehabilitation robot ARMin II in chronic stroke patients: four single-cases. J NeuroEng Rehabil 6(1):46-56

35. Stienen A, Hekman EEG, Prange GB, Jannick M, van der Helm F, van der Kooij H (2009) Dampace: design of an exoskeleton for force-coordination training in upper-extremity rehabilitation. J Med Device 3:031003.1-031003.10

36. Sugar TG, He J, Koeneman EJ, Koeneman JB, Herman R, Huang $\mathrm{H}$, Schultz RS, Herring DE, Wanberg J, Balasubramanian S, Swenson P, Ward JA (2007) Design and control of RUPERT: a device for robotic upper extremity repetitive therapy. Trans Neural Syst Rehabil Eng 15(3):336-346

37. Sunderland A, Tinson DJ, Bradley EL, Fletcher D, Langton HR, Wade DT (1992) Enhanced physical therapy improves recovery of arm function after stroke. A randomised clinical trial. Neurol Neursurg Psychiatry 55:530-535

38. Timmermans AAA, Seelen HAM, Willmann RD, Kingma H (2009) Technology-assisted training of arm-hand skills in stroke: concepts on reacquisition of motor control and therapist guidelines for rehabilitation technology design. J NeuroEng Rehabil $6(1): 1$

39. Tsui CS, Gan JQ, Roberts SJ (2009) A self-paced brain-computer interface for controlling a robot simulator: an online event labelling paradigm and an extended Kalman filter based algorithm for online training. Med Biol Eng Comput 47(3):257-265

40. Wolbrecht E, Chan V, Reinkensmeyer DJ, Bobrow JE (2008) Optimizing compliant, model-based robotic assistance to promote neurorehabilitation. Trans Neural Syst Rehabil Eng 16(3):286-297 